\title{
High-fidelity DNA histograms in neoplastic progression in Barrett's esophagus
}

\author{
Chenggong $\mathrm{Yu}^{1,2}$, Xiaoqi Zhang ${ }^{1,2}$, Qin Huang ${ }^{3,4}$, Michael Klein ${ }^{4,5}$ and Raj K Goyal ${ }^{1,2}$
}

This study describes the high-fidelity DNA histograms in different stages of neoplastic progression to Barrett's adenocarcinoma (BAC). High-fidelity DNA histograms were obtained with image cytometry on sections, and were classified based on DNA index values of the peaks into diploid, mild aneuploid, moderate aneuploid and severe aneuploid. Heterogeneity index $(\mathrm{HI})$ representing cells with different DNA content and the $5 \mathrm{~N}$ exceeding cell fraction were determined. One hundred and eighty-seven cases, including 34 normal gastrointestinal mucosa (control), 66 Barrett'sspecialized intestinal metaplasia (SIM), 22 low-grade dysplasia (LGD), 22 high-grade dysplasia (HGD) and 43 BAC were investigated. Controls showed sharp diploid peaks with HI values less than 13 , and no $5 \mathrm{~N}$ exceeding nuclei. SIM showed a spectrum of histograms including diploid, mild aneuploid and moderate aneuploid histograms. The frequency and severity of aneuploidy increased with worsening histological grades of dysplasia. All BAC cases were aneuploid, with moderate or severe aneuploidy. Marked elevated $\mathrm{HI}$ values $(>20)$ and $5 \mathrm{~N}$ exceeding fractions ( $>5 \%$ ) were found in $5 \%$, $32 \%, 50 \%$ and $88 \%$ of cases with SIM, LGD, HGD and BAC, respectively. The high-fidelity DNA histograms suggest that (1) Barrett's SIM may already be dysplastic in nature, and all BAC may be markedly aneuploid; and (2) elevated cellular DNA heterogeneity and $5 \mathrm{~N}$ fractions may be markers of progressive chromosomal changes and 'unstable aneuploidy' that identifies progressive lesions.

Laboratory Investigation (2007) 87, 466-472. doi:10.1038/labinvest.3700531; published online 19 February 2007

KEYWORDS: Barrett's adenocarcinoma; biomarkers for cancer progression; chromosomal instability; dysplasia; image cytometry; unstable aneuploidy

Barrett's esophagus is an excellent model to study carcinogenesis in humans because it progresses to esophageal carcinoma through a number of clinically recognizable stages of histological dysplasia. ${ }^{1,2}$ However, the role of histological grading of dysplasia in clinical practice is limited because the histological diagnosis is subjective and carries a large inter- and intra-observer variability. ${ }^{1}$ As chromosomal instability may be an important cause of genomic instability leading to Barrett's adenocarcinoma (BAC), DNA aneuploidy has been suggested as a useful marker of neoplastic progression. ${ }^{1,3,4}$ However, currently available studies on DNA ploidy in Barrett's-associated neoplastic lesions use lowfidelity DNA histograms. These studies describe the results only in qualitative terms as diploid or aneuploid. ${ }^{1,4,5}$ Moreover, they miss other details in the DNA histograms that have been shown to be important predictors of progression in other cancers. ${ }^{6,7}$
High-fidelity DNA histograms can provide information on the appearance of the aneuploid clones as well as the cellular DNA content heterogeneity. Appearance of newer clones of severer aneuploidy may indicate disease progression. Several studies in cancers such as breast have shown that increased scatter of cells, including elevation of the $5 \mathrm{~N}$ exceeding fraction, may represent 'unstable aneuploidy' that may be an important marker of progression and prognosticator of clinical outcome. ${ }^{6,7}$

The purpose of the present study was to describe highfidelity DNA histograms obtained by image cytometry on sections (ICS). Our results suggest that Barrett's-specialized intestinal metaplasia (SIM) shows frequent aneuploidy, and frequency and severity of aneuploidy increase with the worsening grades of dysplasia ending in almost universal and severe aneuploidy in BAC. An increasing fraction of cases with SIM, low-grade dysplasia (LGD), high-grade dysplasia

\footnotetext{
'Department of Medicine, VA Boston Healthcare System, Boston, MA, USA; ${ }^{2}$ Harvard Medical School, Boston, MA, USA; ${ }^{3}$ Department of Pathology, VA Boston Healthcare System, Boston, MA, USA; ${ }^{4}$ Brown University School of Medicine, Providence, RI, USA and ${ }^{5}$ VA Providence Healthcare System, Providence, RI, USA Correspondence: Dr RK Goyal, MD, Center for Swallowing and Motility Disorders, VA Boston Healthcare System and Harvard Medical School, 1400 VFW Parkway, Building 3, West Roxbury, MA 02132-4927, USA.

E-mail: raj_goyal@hms.harvard.edu

Received 07 November 2006; revised and accepted 08 January 2007
} 
(HGD), and BAC are found to have markedly elevated cellular DNA heterogeneity and $5 \mathrm{~N}$ exceeding fraction. These cases may represent those of progressive chromosomal abnormalities and the 'unstable aneuploidy'.6,7

We suggest that high-fidelity DNA histograms may be valuable in differentiating normal from neoplastic tissues and the study of neoplastic progression in Barrett's esophagus and many other tumors.

\section{MATERIALS AND METHODS}

The study protocol was approved by the Institutional Review Boards of the Veterans Affairs Boston Healthcare System and the Providence Veterans Affairs Medical Center.

\section{Tissues}

Cases with diagnoses of normal gastrointestinal mucosa, SIM without dysplasia, LGD, HGD and BAC, during the period 1990-2003, were searched in the computer files stored in the Departments of Pathology of the Veterans Affairs Boston Healthcare System and Providence Veterans Affairs Medical Centers. The alcohol formalin-fixed, paraffin-embedded tissue blocks were retrieved.

Two adjacent sections at $7 \mu \mathrm{m}$ were re-cut from the tissue blocks. The sections were hematoxylin and eosin (H\&E) stained for histological evaluation and Feulgen stained for DNA histograms. The 7- $\mu \mathrm{m}$-thick sections provide information on the interphase nuclei. ${ }^{8}$

\section{Histological Evaluation}

Two GI pathologists (QH and MK) reviewed H\&E stained slides independently, according to the published criteria. ${ }^{2,9}$ In cases of disagreement, a consensus diagnosis was reached at the joint microscopic review of the slides in question. Inter-observer disagreement was found in about $25 \%$ of the cases. Barrett's esophagus-related lesions were classified into four categories: Barrett's SIM without dysplasia, LGD, HGD and BAC.

The diagnostic feature of SIM without dysplasia was the goblet cell containing incomplete intestinal metaplasia in the distal esophagus. All these cases had near-normal appearing surface mucosal cells. All cases had some degree of atypia of the crypt cells. ${ }^{2}$ In some cases, there was evidence of ulceration, erosion and mucosal inflammation as evidenced by infiltration by polymorphs or eosinophils. These cases were classified as having reparative atypia. ${ }^{2}$ Cases without ulcerative/inflammatory changes were thought to have non-reparative atypia. ${ }^{10,11}$ All dysplasia cases had dysplastic surface epithelium. Dysplasia was divided into two types: LGD and HGD. In LGD, crypt architecture was largely intact with minimal to mild distortion, but epithelial cells showed evident dysplasia. The nuclei were enlarged, crowded, hyperchromatic, pleomorphic and stratified near the base of the crypts. Characteristic goblet and columnar cell mucus was usually diminished and even absent. Mitotic figures were frequent, particularly in the upper portion of crypts. In
HGD, crypt architecture showed marked disarray, with crypt branching, lateral budding, intra-crypt bridging and complex growth patterns such as papillary-villiforms, and 'backto-back' cribriforms. Nuclear abnormalities in HGD were similar to but more evident than those seen in LGD with marked pleomorphism and stratification reaching the crypt surface, and thus loss of polarization. Mitotic figures were abundant with atypical mitosis. Goblet and columnar cell mucus was usually absent. BAC was diagnosed when malignant cells or abortive glands broke through the basement membrane into the laminar propria or beyond.

\section{DNA Histograms Using ACIS}

DNA histograms on histological sections were obtained using the Automated Cellular Imaging System (ACIS; Clarient Inc, San Juan Capistrano, CA, USA). ${ }^{12}$ Briefly, Feulgen-stained slides were automatically scanned. Only the uniformly stained slides were included. A daily quality control was run using a standard calibration kit from the manufacturer.

The area of interest (area of maximal histological abnormalities) was identified microscopically on H\&E stained sections and marked by a trained pathologist. The corresponding area on the Feulgen-stained section was selected by the operator (CY and XZ) for analysis. ACIS uses a set of image processing algorithms known as Watershed Segmentation to exclude touching nuclei. In these algorithms, nuclei that touch other nuclei are recognized through their size and other morphometric parameters and are separated by insertion of a single pixel-wide boundary at the point of contact. Because Watershed algorithm may cause oversegmentation, particularly in the presence of nuclear overcrowding, manual post-processing correction for merging the oversegmented nuclei was applied. ${ }^{13}$ Moreover, overlapping nuclei, nuclear debris and other artifacts that escaped auto-detection and removal by the system were deleted by the operator.

Non-epithelial (control) cells and the target epithelial cells are imaged, digitized and stored in a file by the system. Around 50 control cells and 200 epithelial cells that are artifact-free are selected. The digital images of nuclei of interest cells were stored individually, and converted into a series of pixels that were quantified as the integrated optical density (IOD) value, representing the DNA content and morphological features of the cells, such as size, shape, contour, granularity and chromatin texture of the nucleus. The mean IOD of control cells is assigned a DNA index (DI) value of 1 , which served as an internal diploid standard and reference for DI calculation of the target epithelial cells. The DI value of 1 corresponds with $2 \mathrm{C}$ or $2 \mathrm{~N}$ (C for copy number of the chromosomes and $\mathrm{N}$ for number of copies of the chromosomes). DNA histograms representing the frequency distribution of the DI values of the population of cells were constructed using the ACIS DNA ploidy software. Digital images and corresponding DI information from the nuclei were stored and the user could easily navigate between 
individual nuclei and their exact position on the DNA histogram.

The coefficients of correlation for two repetitive measurements of the IOD values were greater than 0.9 for both inter- and intra-observer reproducibility. ${ }^{12}$

\section{Analysis of the DNA Histograms}

DNA histograms are plots of DI values $v s$ the number of cells. The presence of peaks of cells with similar DI values and distribution of cells along the histogram were identified. When multiple peaks were present, DI of the most prominent peak was considered. The DI of the peak was determined and the peaks were labeled as diploid (DI 0.9-1.1), mild aneuploid (DI 1.1-1.3), moderate aneuploid (DI 1.3-1.8), or severe aneuploid (DI $>1.8)$. Severe aneuploidy included the histograms with DI between 1.8 and 2.2, which were also labeled tetraploid.

Heterogeneity index (HI) was defined by the number of clusters of cells with IOD at 0.3 intervals in the histograms and it was displayed as different columns (or bars) in the histograms. Fraction of cells with DI exceeding $2.5(5 \mathrm{~N})$ in the histogram was calculated.

\section{Statistical Analysis}

All values are described as mean \pm s.d. One-way betweengroups ANOVA with a trend analysis (ie with groups treated with ascending severity) were performed. A series of nonparametric Kruskal-Wallis tests were also performed. Mean values of two individual groups were compared using post hoc Turkey and Fishers's LSD tests for adjusting the $P$-values, to protect them from multiple comparison effects. Both these tests gave similar results. $\chi^{2}$ test was used to compare frequency distribution of categorical variable. The two-tailed $P$-value of 0.05 or less was considered significant.

\section{RESULTS}

A total of 187 samples from 187 cases (all men) were included in the study, which comprises 34 tissues from normal control gastrointestinal mucosa (control) from colon, duodenum, stomach and esophagus, 66 cases of Barrett's SIM (including 38 cases with reparative atypia and 28 cases with nonreparative atypia), 22 cases of LGD, 22 cases of HGD and 43 cases of BAC.

\section{Patterns of DNA Histograms}

High-fidelity DNA histograms incorporate two important features, namely, DI of the peak and heterogeneity of cells with different DI values including the $5 \mathrm{~N}$ exceeding cellular fraction. Figure 1 showed examples of different patterns of DNA histograms that included a normal diploid histogram with DI of the peak between 0.9 and 1.1 with normal HI (1A); diploid histogram with increased HI (1B); mild aneuploid (paradiploid aneuploidy) with DI of the peak between 1.1 and 1.3 (1C); moderate aneuploidy with DI of the peak between 1.3 and 1.8 (1D); tetraploid aneuploid with the peak
DI between 1.8 and $2.2(1 \mathrm{E})$ and severe aneuploidy with peak DI over 2.2 (1F). Tetraploid aneuploid cases are included in the severe aneuploidy group with peak DI $>1.8$. Note that the main peaks were shorter and broader with worsening aneuploidy. In some cases, multiple overlapping peaks could be appreciated. Markedly elevated cellular DNA heterogeneity and $5 \mathrm{~N}$ exceeding fractions were seen in many aneuploid DNA histograms.

\section{DNA Histograms in Normal Controls and Barrett- Associated Lesions}

\section{Frequency and severity of aneuploidy}

As summarized in Table 1, all normal controls were DNA diploid; 20/66 (31\%) of SIM, 4/22 (18\%) of LGD and 2/22 (9\%) of HGD were also diploid. Diploid histograms were not seen in BAC. Note that majority of cases with SIM 46/66 $(69 \%)$ were aneuploid and the frequency of aneuploidy progressively increased with worsening dysplasia. All BAC cases were aneuploid. The difference in diploidy $v s$ aneuploidy in different groups was significant $(P<0.05)$, except between SIM $v s$ LGD and LGD vs HGD $(P>0.05)$.

The mean DI values of the peaks were significantly different in various groups $(P<0.05)$, except between SIM and LGD. The severity of aneuploidy increased with worsening histological abnormality. Almost two-thirds of SIM and LGD cases with aneuploidy had only mild aneuploidy. In contrast, 15/22 (68\%) of HGD and 41/43 (95\%) of cases with BAC had moderate or severe aneuploidy. Severe aneuploidy was seen in $3 / 22(14 \%)$ of HGD and 18/43 (42\%) of BAC. The difference in the severity of aneuploidy between various groups was statistically significant $(P<0.05)$, except between LGD and HGD $(P>0.05)$. F (linear trend) value $=67.83$; $\mathrm{df}=4$, 182. SIM $<(\mathrm{LGD}=\mathrm{HGD})<$ BAC. One-way betweengroups ANOVA and Kruskal-Wallis ANOVA yielded similar results.

\section{Heterogeneity of the cellular DNA content}

The high-fidelity DNA histograms provided a detailed view of the distribution of cells with different DI values within a histogram. HI was defined by the number of clusters of cells with IOD at 0.3 intervals in the histograms. Mean \pm s.d. values of HI were $9.8 \pm 1.6,16.4 \pm 3.2,20.8 \pm 4.6,23 \pm 6.4$, and $28.9 \pm 7.4$, respectively, in the controls, SIM, LGD, HGD and BAC. These values in various groups were significantly different $(P<0.05)$, except between LGD and HGD $(P>0.05)$.

The upper normal cutoff value of $\mathrm{HI}$ was set at 13 , which represented mean \pm 2 s.d. of the HI in the normal controls. Cases with elevated HI were arbitrarily divided into mild elevation with $\mathrm{HI}$ values between 13 and 20, and severe elevation with $\mathrm{HI}$ value greater than 20 . The results are summarized in Table 2. Note that all normal controls and 12/66 (18\%) of SIM had normal HI. However, almost three-fourths of SIM and almost all cases of dysplasia and BAC had elevated $\mathrm{HI}$. Moreover, the prevalence of severe heterogeneity (HI > 20) increased from 6/66 (10\%) in SIM to 10/22 (46\%) 
a

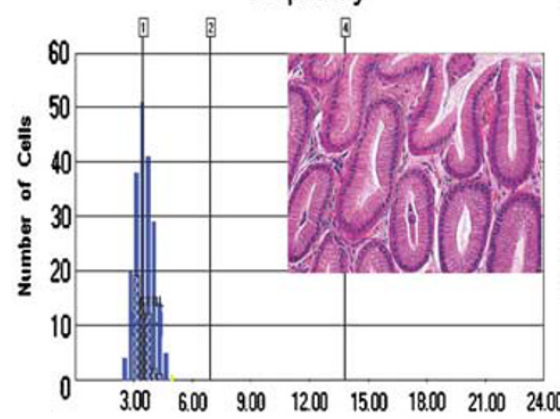

d

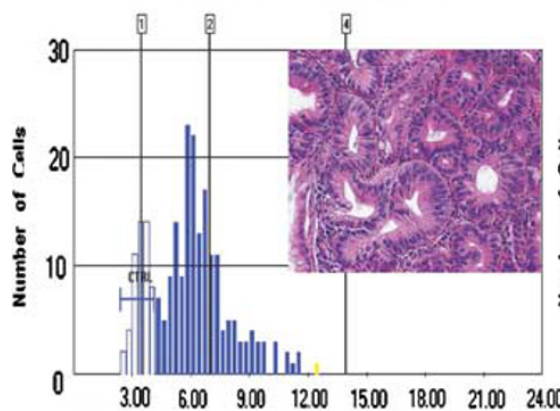

b

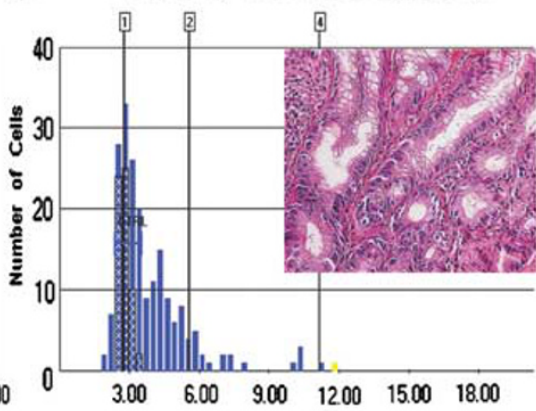

e Severe aneuploidy(Tetraploidy)

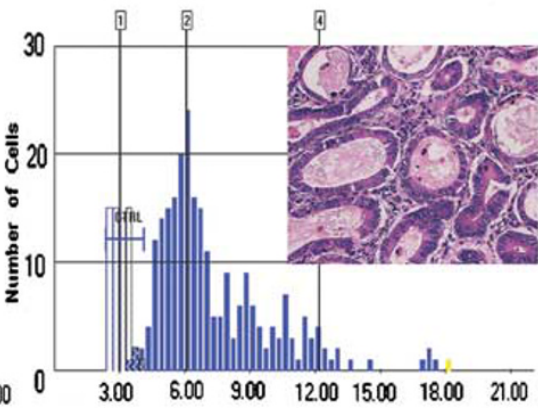

c

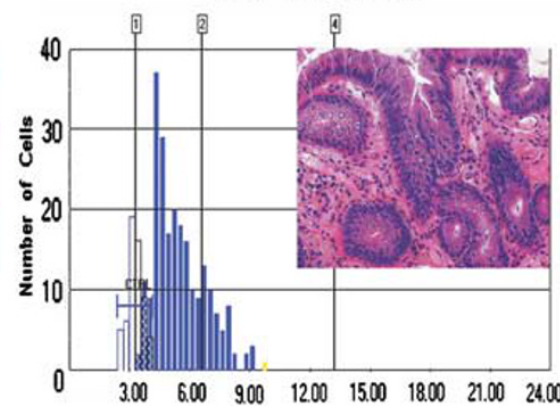

f

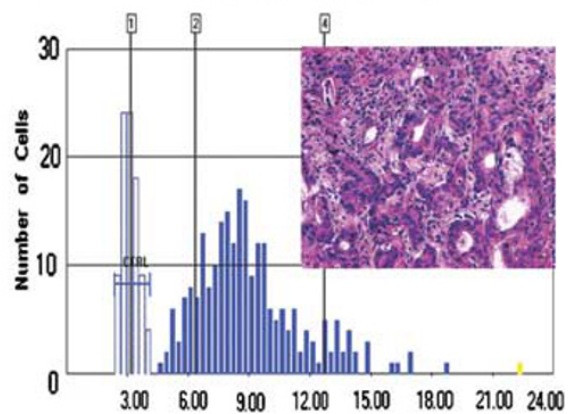

Figure 1 Patterns of DNA histograms seen on ICS in Barrett's-associated mucosal lesions. The image of the corresponding H\&E-stained sections was shown

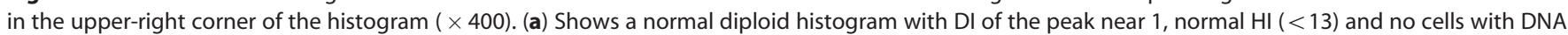
$>5 \mathrm{~N}$. (b) Shows a diploid histogram with peak DI near 1, increased $\mathrm{HI}$ of 23 and $5.1 \%$ cells with DNA $>5 \mathrm{~N}$. This pattern has been described as diploid peak with right skewing. (c) Shows absence of a diploid peak that is replaced by an aneuploid peak with DI 1.3. This pattern represents mild aneuploidy or paradiploid aneuploidy. Also note elevated $\mathrm{HI}$ of 20 and $2.6 \% 5 \mathrm{~N}$ exceeding fraction. (d) Shows absence of a diploid peak and presence of a broad aneuploid peak with DI of 1.7. This pattern is classified as moderate aneuploid. Also note an elevated $\mathrm{HI}$ of 24 and $5 \mathrm{~N}$ exceeding rate of $12.2 \%$. (e) Shows absence of a diploid peak that is replaced by a broad aneuploid peak with DI of 2.01. This pattern, with peak DI between 1.8 and 2.2, was called tetraploid or tetraploid aneuploid. Tetraploid pattern is a form of severe aneuploidy. Note that this histogram has a large $\mathrm{HI}$ of 39 and $33.6 \%$ cells with DI greater than $5 \mathrm{~N}$. (f) Shows that there is no diploid peak. The aneuploid peak is broad and has a DI value of 2.7.This group includes all case with peak DI $>2.2$ and they are called severe aneuploid. Also note this histogram has very elevated $\mathrm{HI}$ of 39 . Most of the peak has DI values greater than $5 \mathrm{~N}$. $63.9 \%$ of cell in this histograms have DI values greater than $5 \mathrm{~N}$. Normal diploid pattern was seen in all normal controls and some SIM cases. Diploid with increased heterogeneity was seen mostly in SIM and LGD; mild aneuploidy was seen in all histological types, but most frequently in SIM and LGD; moderated aneuploidy was seen in all histological types, but most commonly in HGD and BAC; and severe aneuploidy was seen primarily in HGD and BAC cases.

in LGD, $12 / 22$ (54\%) in HGD and $41 / 43$ (95\%) in BAC. The groups differed significantly from each other regarding the degree of $\mathrm{HI}$ elevation $(P<0.05)$, except LGD and HGD $(P>0.05)$. F (linear trend) value $=82.6$; $\mathrm{df}=4,182$. SI$\mathrm{M}<(\mathrm{LHD}=\mathrm{HGD})<\mathrm{BAC}$. One-way between-groups ANOVA and Kruskal-Wallis ANOVA yielded similar results.

\section{$5 \mathrm{~N}$ exceeding fraction}

$5 \mathrm{~N}$ exceeding fraction is a component of the HI. The increased scatter of cells with DI in the S phase (DI values between 1.1 and 1.8) and G2 phase (DI values between 1.8 and 2.2) of the cell cycle may be because of increased proliferation. However, cells with DNA content $>5 \mathrm{~N}$ cannot be explained by increased normal proliferation of diploid cells with G0/G1 peak. Therefore, we determined the $5 \mathrm{~N}$ exceeding fraction. The cases were divided into normal $(<1 \%$ cells), slightly abnormal ( $1-5 \%$ cells) and severely abnormal ( $>5 \%$ cells) $5 \mathrm{~N}$ exceeding fractions. As shown in Table 3, normal gastrointestinal control cells had no cell with DI exceeding 5N. Prevalence of severely abnormal $5 \mathrm{~N}$ exceeding fraction progressively increased from $3 / 22(5 \%)$ in SIM to $7 / 22(32 \%)$ in LGD, 12/22 (54\%) in HGD and 38/43 (88\%) in BAC. The groups differed significantly in regards to the degree of $>5 \mathrm{~N}$ fraction $(P<0.05)$ except SIM and LGD. $\mathrm{F}$ (linear trend) value $=42.96 ; \mathrm{df}=3,149(\mathrm{SIM}=\mathrm{LGD})<$ HGD $<$ BAC. Because the Turkey test only describes the differences at 0.5 level only, we further compared mean values of SIM and LGD using Fisher's LSD test, which yielded a $P$-value of 0.07 , suggesting a tendency for these two groups to differ.

\section{Relationship between the degree of aneuploidy, $\mathrm{HI}$ and $5 \mathrm{~N}$} exceeding fraction

Severity of aneuploidy correlated well with the degree of heterogeneity (Table 4 ). The prevalence of severely elevated HI $(>20)$ was $1 / 60(2 \%)$ in diploid, 9/50 (18\%) in mildly aneuploid, 38/56 (68\%) in moderately aneuploid and 21/21 $(100 \%)$ in severe aneuploid histograms $(P<0.05$ in different 
Table 1 Severity of aneuploidy in various Barrett's-associated histological lesions

\begin{tabular}{lcccccc}
\hline Groups & $n$ & Diploidy (\%) & \multicolumn{5}{c}{ Aneuploidy (\%) } \\
\cline { 4 - 7 } & & & Total & Mild & Moderate & Severe \\
\hline Control & 34 & $34(100)$ & 0 & 0 & 0 & 0 \\
SIM & 66 & $20(31)$ & $46(69)$ & $32(48)$ & $14(21)$ & 0 \\
LGD & 22 & $4(18)$ & $18(82)$ & $11(50)$ & $7(32)$ & 0 \\
HGD & 22 & $2(9)$ & $20(91)$ & $5(23)$ & $12(54)$ & $3(14)$ \\
BAC & 43 & 0 & $43(100)$ & $2(5)$ & $23(53)$ & $18(42)$ \\
& & & & & & \\
Total & 187 & 60 & 127 & 50 & 56 & 21 \\
\hline
\end{tabular}

The groups differed significantly in ploidy status $(P<0.05)$, except SIM vs LGD and LGD vs HGD $(P>0.05)$. The groups also differed significantly in the severity of aneuploidy $(P<0.05)$ except LGD and HGD $(P>0.05)$.

Table 2 Distribution of cases with different grades of cellular DNA content heterogeneity index in different Barrett'sassociated histological lesions

\begin{tabular}{lcccc}
\hline Groups & $\mathrm{n}$ & $\leq 13(\%)$ & $14-20(\%)$ & $>20(\%)$ \\
\hline Control & 34 & $34(100)$ & 0 & 0 \\
SIM & 66 & $12(18)$ & $48(72)$ & $6(10)$ \\
LGD & 22 & 0 & $12(54)$ & $10(46)$ \\
HGD & 22 & $1(5)$ & $9(41)$ & $12(54)$ \\
BAC & 43 & 0 & $2(5)$ & $41(95)$ \\
& & & & 69 \\
Total & 187 & 47 & 71 & 69 \\
\hline
\end{tabular}

The groups differed significantly with regard to the degree of $\mathrm{HI}$ elevation $(P<0.05)$, except between LGD and HGD $(P>0.05)$.

groups). Similarly, $5 \mathrm{~N}$ exceeding fraction rates $<5 \%$ and $>5 \%$ were also significantly different in different groups $(P<0.05)$.

There was a strong correlation among peak DI, HI and $5 \mathrm{~N}$ exceeding fraction. The colinearity had scores ranging from 0.74 (peak DI and $\mathrm{HI}$ ) to 0.87 ( $\mathrm{HI}$ and $5 \mathrm{~N}$ exceeding fraction).

Overall, normal DNA histogram as established by findings in normal control gastrointestinal mucosal cells was characterized by diploid peak with DI between 0.9-1.1, DNA content $\mathrm{HI}$ of $<13$ and no cell with DI exceeding $5 \mathrm{~N}$.

Barrett's SIM showed a variety of different histograms. A total of 6/66 (9\%) cases showed normal DNA diploid histograms without increased DNA content heterogeneity; 14/66 (22\%) showed DNA diploid histograms with elevated HI ( $>13$ ) (out of these one case had $>5 \% 5 \mathrm{~N}$ exceeding fraction); 46/66 (69\%) were aneuploid with mild aneuploidy
Table 3 Distribution of cells with $5 \mathrm{~N}$ exceeding rate in different Barrett's-associated histological lesions

\begin{tabular}{lcccc}
\hline Groups & $\mathrm{n}$ & $\leq 1(\%)$ & $1-5(\%)$ & $>5(\%)$ \\
\hline Control & 34 & $34(100)$ & 0 & 0 \\
SIM & 66 & $52(79)$ & $11(16)$ & $3(5)$ \\
LGD & 22 & $8(36)$ & $7(32)$ & $7(32)$ \\
HGD & 22 & $5(23)$ & $5(23)$ & $12(54)$ \\
BAC & 43 & $1(3)$ & $4(9)$ & $38(88)$ \\
& & & & 60 \\
Total & 187 & 100 & 27 & \\
\hline
\end{tabular}

The groups differed significantly with regard to the degree of $5 \mathrm{~N}$ exceeding rates $(P<0.05)$, except between SIM and LGD $(P>0.05)$.

Table 4 Relationship between presence and severity of aneuploidy and heterogeneity index and $5 \mathrm{~N}$ exceeding fraction

\begin{tabular}{lccccc}
\hline Groups & $\mathrm{n}$ & $\leq 20(\%)$ & $>20(\%)$ & $\leq 5(\%)$ & $>5(\%)$ \\
\hline Diploid & 60 & $59(98)$ & $1(2)$ & $59(98)$ & $1(2)$ \\
Mild aneuploid & 50 & $41(82)$ & $9(18)$ & $46(92)$ & $4(8)$ \\
Moderate aneuploid & 56 & $18(32)$ & $38(68)$ & $22(39)$ & $34(61)$ \\
Severe aneuploid & 21 & 0 & $21(100)$ & 0 & $21(100)$ \\
Total & 187 & 118 & 69 & 127 & 60 \\
\hline
\end{tabular}

The DNA histograms showing diploidy and various severities of aneuploidy differed in regard to normal or mildly elevated $\mathrm{HI}$ vs severely elevated $(P<0.01)$. They also differed significantly with regard to normal or mildly $(<5 \%)$ and severely $(>5 \%)$ elevated $5 \mathrm{~N}$ exceeding fractions $(P<0.05)$.

in 32/66 (48\%) and moderate aneuploidy in 14/66 (21\%). No case had severe aneuploidy. 6/66 (10\%) of SIM had severe DNA content heterogeneity and $3 / 66(5 \%)$ had marked elevation of $5 \mathrm{~N}$ exceeding fraction. Both severely elevated $\mathrm{HI}$ and $5 \mathrm{~N}$ exceeding rate were seen in $3 / 66(5 \%)$ of SIM.

There was no difference in peak DI values in groups of cases with reparative atypia or non-reparative atypia. The peak DI was $1.22 \pm 0.15$ in cases with reparative atypia $(n=38)$ and $1.20 \pm 0.12$ in cases with non-reparative atypia $(n=28)(P>0.05)$.

LGD cases showed similar to but more frequent and severe abnormalities than SIM. No case showed normal diploid DNA histogram; 4/22 (18\%) showed diploidy with elevated heterogeneity; 11/22 (50\%) were mildly aneuploid; 7/22 (32\%) were moderately aneuploid; no case had severe aneuploidy. All cases had increased heterogeneity with 10/22 (46\%) having marked heterogeneity and 7/22 (32\%) having markedly elevated $5 \mathrm{~N}$ exceeding fraction. Both severely elevated $\mathrm{HI}$ and $5 \mathrm{~N}$ exceeding rate were seen in $7 / 22(32 \%)$ of the cases. 
HGD cases showed more frequent and severer abnormalities than LGD. No case showed a diploid DNA histogram; 2/22 (9\%) showed diploidy with elevated heterogeneity; 5/22 (23\%) were mildly aneuploid; $12 / 22$ (54\%) were moderately aneuploid; and 3/22 (14\%) were severely aneuploidy. All cases had increased heterogeneity with 12/22 (54\%) having marked heterogeneity and 12/22 (54\%) having markedly elevated $5 \mathrm{~N}$ exceeding fraction. Both severely elevated $\mathrm{HI}$ and $5 \mathrm{~N}$ exceeding rate were seen in $11 / 22(50 \%)$ of the cases.

BAC cases showed the severest DNA content abnormalities. No case showed a diploid DNA histogram; 2/43 (5\%) were mildly aneuploid, 23/43 (53\%) were moderately aneuploid and 18/43 (42\%) were severely aneuploidy. All cases had increased heterogeneity with 41/43 (95\%) having marked heterogeneity and 38/43 (88\%) having both markedly elevated $\mathrm{HI}$ and $5 \mathrm{~N}$ exceeding fraction.

\section{DISCUSSION}

This study shows that (1) high-fidelity DNA histograms provide quantitative information on the presence and the degree of aneuploidy, cellular DNA content heterogeneity and $5 \mathrm{~N}$ exceeding fractions; (2) the DNA histograms in SIM are frequently abnormal; (3) most of the BAC have moderate or severe aneuploidy, and frequency and grade of aneuploidy increases with worsening histological grades of histological dysplasia; (4) severely elevated DNA content heterogeneity and $5 \mathrm{~N}$ exceeding fraction (indicating unstable aneuploidy) are seen in a few cases of SIM, many case with dysplasia and most cases of BAC.

Accuracy and fidelity of the DNA histogram depends on several factors. One of the important factors in obtaining reliable DNA histograms is the proper selection of the test sample. DNA histograms obtained with standard flow cytometry (FC) suffer from inclusion of unknown quantities of the non-epithelial cells in the test sample. In recent years, attempts at better definition of the sample to be tested by FC have included labeling of the cells of interest ${ }^{14}$ or selecting laser dissected areas of interest. In ICS, the selected test sample includes visually identified the epithelial cells in an area of interest. The area of interest is marked on the H\&Estained slide by a pathologist and the technician investigates all the artifact-free epithelial cells in that area, thereby a selection bias by the technician is eliminated.

The fidelity of the DNA histogram depends on the technique of cytometry. DNA histograms on FC focus on major peaks of cells and overlook minor cell groups or single cells with different DNA contents, and thereby underestimate DNA content heterogeneity and $5 \mathrm{~N}$ exceeding fraction of cells. ${ }^{15,16}$ ICS may also miss rare events including the cells exceeding $5 \mathrm{~N}$, unless special software is used. ${ }^{7}$ ICS using ACIS provided high-fidelity DNA histograms that defined presence and degree of aneuploidy, cellular DNA content heterogeneity and $5 \mathrm{~N}$ exceeding fractions.
Increased cellular DNA heterogeneity with a diploid G0/ G1 peak may result from elevated S- or G2-phase fractions associated with increased normal proliferation. However, cellular DNA heterogeneity associated with aneuploid G0/G1 peak itself may result from elevated heterogeneity of the cells in the G0/G1 phase of the cell cycle or overlapping clones of new aneuploid cells. We found that severely elevated cellular DNA heterogeneity is associated with severely elevated $5 \mathrm{~N}$ exceeding fraction. The $5 \mathrm{~N}$ exceeding fraction in the interphase nuclei is always abnormal and may represent: (1) aneuploid cells in S or G2 phases; (2) severely aneuploid cells in G0/G1 phase; (3) diploid cells in second S phase during endoreplication; (4) product of diploid cells with unbalanced anaphase including G2 phase of the larger segment and second S phase of the smaller segment. More recently, studies in breast cancer have shown that the so-called 'scatter index' that represents cellular DNA content heterogeneity and $5 \mathrm{~N}$ exceeding fraction on high-fidelity DNA histograms may be markers of increased chromosomal instability and worse clinical outcome. ${ }^{6,17}$

High-fidelity DNA histograms showed that the DNA content abnormalities in SIM are far more common than appreciated previously by standard FC. ${ }^{18}$ This study found that majority $(46 / 66,69 \%)$ of the cases with SIM was aneuploid. Moreover, almost one-half of the SIM cases with diploid histogram had elevated heterogeneity index. The aneuploid cases were only mildly or moderately aneuploid. Interestingly, HI was elevated in majority $(54 / 66,82 \%)$ of the SIM cases. These observations suggested that elevation of heterogeneity might be an early abnormality in SIM. A small number $(3 / 66,5 \%)$ of SIM also showed aneuploidy with severely elevated $\mathrm{HI}$ and $5 \mathrm{~N}$ exceeding fraction; these cases may represent cases of unstable aneuploidy and progressive lesions. The high incidence of DNA histogram abnormality in SIM in high-fidelity histograms is consistent with the finding of high incidence of chromosomal changes in Barrett's SIM without dysplasia. Walch and co-workers reported that there was an average of seven chromosomal imbalances per case in SIM. ${ }^{19}$ These studies suggest that, although called metaplasia, SIM should in fact be considered as dysplasia. ${ }^{19}$ All cases with non-dysplastic SIM showed some degree of atypia. ${ }^{2}$ The non-dysplastic SIM cases included cases that may be considered as having reparative crypt cell atypia. Such cases were identified by the presence of ulceration, erosion or inflammatory changes. ${ }^{2}$ We found no difference in the mean peak DI content of nuclei in cases with reparative atypia or non-reparative atypia. Further studies are needed to determine whether repair or inflammatory processes per se are associated DNA content abnormalities. ${ }^{16}$

The term 'dysplasia' implies a premalignant neoplasm that is an intermediate stage between normal epithelium and cancer. It is usually defined morphologically, however, histological grading of dysplasia is in part arbitrary and subjective and, therefore, suffers from a high-degree of inter- and intra-observer variability. ${ }^{20}$ Steinbeck and co-workers have 
suggested that dysplasia may be better defined by the DNA aneuploidy determination. ${ }^{16}$ This view is supported by the observations that hyperplasia that may resemble LGD on histology, can be distinguished from LGD by DNA histograms. ${ }^{16}$ Moreover, DNA ploidy analysis has the advantage of defining dysplasia at a mechanistic level and providing objective results. Studies are needed to determine whether high-fidelity DNA histogram and histological evaluation together may provide a better measure of severity of dysplasia. Morphological studies have suggested that a fraction of cases with LGD and HGD progress to BAC. ${ }^{18,21}$ It is possible that the presence of unstable aneuploidy in the DNA histogram may identify cases of LGD or HGD that are at risk of progression to cancer. Planned prospective studies are needed to test this hypothesis.

High-fidelity DNA histograms also showed that all the BAC investigated had moderate or severe aneuploid. These results are different from studies that used standard FC to detect aneuploidy. By standard FC, only about half to twothirds (40-69\%) of the cases of BAC have been reported to be aneuploid. ${ }^{22,23}$ This difference may be due to a high false negative rate for FC in diagnosing aneuploidy. Several studies in other carcinomas such as breast have reported that many cancers that were reported to be diploid on standard FC were found to be aneuploid by image cytometry. ${ }^{24}$ Cellular DNA heterogeneity, scatter index or elevated $5 \mathrm{~N}$ exceeding fraction in BAC were not reported in earlier studies using low-fidelity DNA histograms. The high-fidelity DNA histograms revealed that a majority $(38 / 43,88 \%)$ of the cases of BAC had both severely elevated $\mathrm{HI}$ and $5 \mathrm{~N}$ exceeding fraction. These cases may represent cases with unstable aneuploidy that mark progressive cancers. The very high rate of unstable aneuploidy in BAC may be consistent with its progressive nature and poor clinical outcome. ${ }^{1}$

In conclusion, ICS provides high-fidelity DNA histograms that provide precise information on the presence and the degree of aneuploidy, cellular DNA content heterogeneity and $5 \mathrm{~N}$ exceeding fraction. These studies show that Barrett's SIM has a high prevalence of DNA aneuploidy which is consistent with its neoplastic nature. BAC has a very high prevalence of severe unstable aneuploidy that is consistent with the highly aggressive nature of this cancer. ${ }^{1}$ Cases with SIM and dysplasia have progressively increasing frequency and severity of aneuploidy and 'unstable aneuploidy' that may serve as clinical markers of progression to cancer.

\section{ACKNOWLEDGEMENT}

We thank Errol Baker (PhD) for advice and help with statistical analysis, and Jonathan Burry (MD) and Hashem El-Serag (MD) for helpful comments.

Source of support: This work was supported in part by NIH grants DK62867 and Merit Review Award from the Research Service, Veterans Health Administration. Chenggong Yu and Xiaoqi Zhang were supported in part by Affiliated Nanjing Drum Tower Hospital of Nanjing University School of Medicine, PR China.

\section{DUALITY OF INTEREST}

The authors have no duality of interest to declare.

1. Koppert LB, Wijnhoven BP, van Dekken $\mathrm{H}$, et al. The molecular biology of esophageal adenocarcinoma. J Surg Oncol 2005;92:169-190.

2. Goldblum JR. Barrett's esophagus and Barrett's-related dysplasia. Mod Pathol 2003;16:316-324.

3. Rajagopalan H, Lengauer C. Aneuploidy and cancer. Nature 2004; 432:338-341.

4. Reid BJ, Blount PL, Rabinovitch PS. Biomarkers in Barrett's esophagus. Gastrointest Endosc Clin N Am 2003;13:369-397.

5. Fang $M$, Lew $E$, Klein $M$, et al. DNA abnormalities as marker of risk for progression of Barrett's esophagus to adenocarcinoma: image cytometric DNA analysis in formalin-fixed tissues. Am J Gastroenterol 2004;99:1887-1894.

6. Kronenwett U, Huwendiek S, Ostring C, et al. Improved grading of breast adenocarcinomas based on genomic instability. Cancer Res 2004;64:904-909.

7. Lorenzato M, Abboud $P$, Masure $M$, et al. Image cytometry detection of breast cancer cells with > 5C DNA content and minor DNA stemlines. Anal Quant Cytol Histol 2000;22:199-205.

8. Steinbeck RG, Auer GU, Zetterberg AD. Reliability and significance of DNA measurements in interphase nuclei and division figures in histological sections. Eur J Cancer 1999;35:787-795.

9. Haggitt RC. Barrett's esophagus, dysplasia, and adenocarcinoma. Hum Pathol 1994;25:982-993.

10. Poulin N, Susnik B, Guillaud M, et al. Histometric texture analysis of DNA in thin sections from breast biopsies. Application to the detection of malignancy-associated changes in carcinoma in situ. Anal Quant Cytol Histol 1995;17:291-299.

11. Reid BJ, Haggitt RC, Rubin CE, et al. Observer variation in the diagnosis of dysplasia in Barrett's esophagus. Hum Pathol 1988;19:166-178.

12. Huang $Q, Y u C$, Klein $M$, et al. DNA index determination with Automated Cellular Imaging System (ACIS) in Barrett's esophagus: comparison with CAS 200. BMC Clin Pathol 2005;5:7.

13. Pham DL, Xu C, Prince JL. Current methods in medical image segmentation. Annu Rev Biomed Eng 2000;2:315-337.

14. Rickes S, Hauptmann S, Flath B, et al. Development of a flow cytometric method to determine DNA ploidy of oesophageal cancer cells obtained by forceps biopsy samples during oesophagogastro-duodenoscopy. Onkologie 2003;26:32-37.

15. Auer G, Askensten U, Ahrens O. Cytophotometry. Hum Pathol 1989:20:518-527.

16. Steinbeck RG. Dysplasia in view of the cell cycle. Eur J Histochem 2004:48:203-211.

17. Lorenzato M, Caudroy S, Bronner C, et al. Cell cycle and/or proliferation markers: what is the best method to discriminate cervical high-grade lesions? Hum Pathol 2005;36:1101-1107.

18. Reid BJ, Levine DS, Longton G, et al. Predictors of progression to cancer in Barrett's esophagus: baseline histology and flow cytometry identify low- and high-risk patient subsets. Am J Gastroenterol 2000;95:1669-1676.

19. Walch AK, Zitzelsberger HF, Bruch J, et al. Chromosomal imbalances in Barrett's adenocarcinoma and the metaplasia-dysplasia-carcinoma sequence. Am J Pathol 2000;156:555-566.

20. Montgomery E, Bronner MP, Goldblum JR, et al. Reproducibility of the diagnosis of dysplasia in Barrett esophagus: a reaffirmation. Hum Pathol 2001;32:368-378.

21. Schnell TG, Sontag SJ, Chejfec G, et al. Long-term nonsurgical management of Barrett's esophagus with high-grade dysplasia. Gastroenterology 2001;120:1607-1619.

22. Flejou JF, Doublet B, Potet F, et al. DNA ploidy in adenocarcinoma of Barrett's esophagus. Ann Pathol 1990;10:161-165.

23. Reid BJ, Sanchez CA, Blount PL, et al. Barrett's esophagus: cell cycle abnormalities in advancing stages of neoplastic progression. Gastroenterology 1993;105:119-129.

24. Alanen KA, Lintu M, Joensuu $\mathrm{H}$. Image cytometry of breast carcinomas that are DNA diploid by flow cytometry: time to revise the concept of DNA diploidy? Anal Quant Cytol Histol 1998;20:178-186. 